\title{
Delegate list
}

\begin{tabular}{|c|c|c|}
\hline Name & Job title & Workplace \\
\hline Rhona Arthur & Assistant Director & CILIPS/SLIC \\
\hline Chris Awre & $\begin{array}{l}\text { Integration Architect and CREE } \\
\text { Project Manager }\end{array}$ & University of Hull \\
\hline Deborah Ball (afternoon only) & Research and Data Officer & Lifelong Learning UK \\
\hline Chris Batt & Chief Executive & $\begin{array}{l}\text { Museums Libraries and } \\
\text { Archives Council }\end{array}$ \\
\hline David Bawden & $\begin{array}{l}\text { Editor, Journal of } \\
\text { Documentation }\end{array}$ & City University London \\
\hline Andrew Booth & $\begin{array}{l}\text { Director of Information } \\
\text { Resources }\end{array}$ & University of Sheffield \\
\hline Richard Boulderstone & $\begin{array}{l}\text { Director of e-strategy and } \\
\text { Information Systems }\end{array}$ & British Library \\
\hline Caroline Brazier & $\begin{array}{l}\text { Head, Collection Acquisitions } \\
\text { and Description }\end{array}$ & British Library \\
\hline Lynne Brindley & Chief Executive & British Library \\
\hline Peter Brophy & Director of CERLIM, & $\begin{array}{l}\text { Manchester Metropolitan } \\
\text { University }\end{array}$ \\
\hline Rachel Bruce & $\begin{array}{l}\text { Programme Director } \\
\text { (Information Environment) }\end{array}$ & King's College London \\
\hline Stephen Bury & $\begin{array}{l}\text { Head, European and American } \\
\text { Collections }\end{array}$ & British Library \\
\hline Terry Cannon & & British Library \\
\hline Mel Collier & $\begin{array}{l}\text { Director of the University } \\
\text { Library }\end{array}$ & Catholic University of Leuven \\
\hline Ellen Collins & Research and Policy Assistant & $\begin{array}{l}\text { Museums Libraries Archives, } \\
\text { London }\end{array}$ \\
\hline Louise Cooke & $\begin{array}{l}\text { Lecturer, Library Information } \\
\text { Management }\end{array}$ & Loughborough University \\
\hline Carl Cross & Young People's Librarian & Derbyshire County Council \\
\hline Judith Elkin & $\begin{array}{l}\text { Pro Vice Chancellor and Deputy } \\
\text { Chief Executive }\end{array}$ & University of Worcester \\
\hline Juliet Eve & $\begin{array}{l}\text { Senior Lecturer, Division of } \\
\text { Information and Media Studies }\end{array}$ & University of Brighton \\
\hline Gayner Eyre & $\begin{array}{l}\text { Head of Department of } \\
\text { Information Studies }\end{array}$ & University of Wales Aberystwyth \\
\hline John Feather & $\begin{array}{l}\text { Professor of Library and } \\
\text { Information Studies }\end{array}$ & Loughborough University \\
\hline Biddy Fisher & Head of Information Studies & Sheffield Hallam University \\
\hline
\end{tabular}




\begin{tabular}{|c|c|c|}
\hline Name & Job title & Workplace \\
\hline Rob Froud & Head of Cultural Service & Somerset County Council \\
\hline Cathryn Gallacher & $\begin{array}{l}\text { Assistant Director of } \\
\text { Information Services }\end{array}$ & University of Bristol \\
\hline $\begin{array}{l}\text { Chitro Ghose } \\
\text { (morning only) }\end{array}$ & Research and Data Manager & Lifelong Learning UK \\
\hline Anne Goulding & $\begin{array}{l}\text { Editor, Journal of Information } \\
\text { Science }\end{array}$ & Loughborough University \\
\hline June Hedges & $\begin{array}{l}\text { Co-ordinator, Teaching and } \\
\text { Learning Support section }\end{array}$ & UCL Library Services \\
\hline Debbie Hicks & Director, Research and Strategy & Reading Agency \\
\hline Linda Houston & Executive Officer & LISC (NI) \\
\hline Elspeth Hyams & $\begin{array}{l}\text { Editor, Library and Information } \\
\text { Update }\end{array}$ & CILIP \\
\hline Michael Jubb & Director & Research Information Network \\
\hline Stephanie Kenna & $\begin{array}{l}\text { Manager, Regional and Library } \\
\text { Programmes }\end{array}$ & British Library \\
\hline Derek Law & $\begin{array}{l}\text { Librarian and Head of } \\
\text { Information Resources } \\
\text { Directorate }\end{array}$ & University of Strathclyde \\
\hline Martin Lewis & $\begin{array}{l}\text { Director of Library Services and } \\
\text { University Librarian }\end{array}$ & University of Sheffield \\
\hline Liz Lyon & Director, UKOLN & University of Bath \\
\hline Nigel Macartney & Director of Information Services & University of Ulster \\
\hline Mala Mann & $\begin{array}{l}\text { Information Specialist, Support } \\
\text { Unit for Research Evidence }\end{array}$ & Cardiff University \\
\hline Pat Manson & $\begin{array}{l}\text { Head of Unit, Learning and } \\
\text { Cultural Heritage }\end{array}$ & European Commission \\
\hline Bob McKee & Chief Executive & CILIP \\
\hline Matthew Mezey & $\begin{array}{l}\text { News Editor, Library and } \\
\text { Information Update }\end{array}$ & CILIP \\
\hline Martin Molloy & $\begin{array}{l}\text { Director of Cultural and } \\
\text { Community Services }\end{array}$ & Derbyshire County Council \\
\hline William Nixon & Project Manager, DAEDALUS & Glasgow University Library \\
\hline Caroline Oades & Head of Research & $\begin{array}{l}\text { Associated Chartered Certified } \\
\text { Accountants }\end{array}$ \\
\hline Charles Oppenheim & $\begin{array}{l}\text { Professor of Information } \\
\text { Services and Head of } \\
\text { Department }\end{array}$ & Loughborough University \\
\hline Miggie Pickton & $\begin{array}{l}\text { Academic Librarian, Information } \\
\text { Services }\end{array}$ & University of Northampton \\
\hline Malcolm Read & Executive Secretary & JISC \\
\hline
\end{tabular}




\begin{tabular}{|c|c|c|}
\hline Name & Job title & Workplace \\
\hline Seamus Ross & $\begin{array}{l}\text { Director, Humanities Advanced } \\
\text { Technology and Information } \\
\text { Institute }\end{array}$ & University of Glasgow \\
\hline Noeleen Schenk & Consultant & \\
\hline Seamus Simpson & $\begin{array}{l}\text { Principal Lecturer and Research } \\
\text { Co-ordinator, Department of } \\
\text { Information and } \\
\text { Communications }\end{array}$ & $\begin{array}{l}\text { Manchester Metropolitan } \\
\text { University }\end{array}$ \\
\hline Pat Spoor & Faculty Team Leader (Health) & University of Leeds \\
\hline Michael Stead & e-Resources Librarian & $\begin{array}{l}\text { Bolton, Trafford and Wigan } \\
\text { Libraries }\end{array}$ \\
\hline Jan Suchacki & $\begin{array}{l}\text { Learning and Access Policy } \\
\text { Adviser }\end{array}$ & $\begin{array}{l}\text { Museums Libraries Archives, } \\
\text { South West }\end{array}$ \\
\hline Lucy Tedd & Editor, Program & University of Wales Aberystwyth \\
\hline Briony Train & $\begin{array}{l}\text { Lecturer in Librarianship and } \\
\text { MA Librarianship Programme } \\
\text { Coordinator }\end{array}$ & University of Sheffield \\
\hline Jan Wilkinson & Head of Higher Education & British Library \\
\hline Peter Willett & Professor of Information Science & University of Sheffield \\
\hline Martin Wolf & Social Services Librarian & University of Warwick \\
\hline Helen Young & Academic Librarian & Loughborough University \\
\hline
\end{tabular}

